\title{
EDUCATION ET DÉMOCRACIE
}

\section{(EDUCATION AND DEMOCRACY)}

BERNARD HUGONNIER ${ }^{1}$

Institut Catholique de Paris

\section{RÉSUMÉ}

Cet article s'adresse à trois questions : quelle est la relation précise entre démocratie et éducation dans les temps présents ? Que nous apprennent les résultats de la dernière enquête internationale PISA sur la situation de la démocratie dans les pays les plus avancés (soit l'Occident) ? Enfin, que nous révèlent les récents résultats des élections aux Etats-Unis, de nouveau, sur la situation de la démocratie mais aussi sur les besoins en termes d'éducation ? L'analyse montre que, dans un temps où les économies sont mondialisées, et à l'ère de l'économie de la connaissance et du numérique, il faut repenser le rôle que doit jouer l'éducation vis-à-vis de la démocratie. L'analyse montre aussi qu'il y a un risque de recul de la démocratie dans les pays occidentaux dû à l'augmentation du nombre de jeunes ayant une très faible éducation et à une diminution de ceux en ayant une excellente (c'est-à-dire l'élite). Enfin, le papier établit que face à une montée du nationalisme et d'un certain totalitarisme (notamment aux Etats-Unis), élever le niveau d'éducation de la population est certainement la meilleure réponse à apporter.

Mots clés : Éducation à la citoyenneté, démocratie, autonomie et esprit critique.

\section{ABSTRACT}

This article addresses three questions: what is the precise relationship between democracy and education in the present times? What do we learn from the results of the latest international PISA survey on the state of democracy in the most advanced countries (the West)? Finally, what do the recent results of the elections in the United States reveal about the situation of democracy but also the needs in terms of education? The analysis shows that, at a time when economies are globalized and in the era of the knowledge and digital economy, it is necessary to rethink the role that education must play in relation to democracy. The analysis also shows that there is a risk of a decline in democracy in Western countries due to an increase in the number of young people with very low education and a decrease in those with an excellent education (i.e. the elite). Finally, the paper states that, in the face of rising nationalism and totalitarianism (particularly in the United States), raising the level of education of the population is certainly the best answer to be given.

Key words: Citizenship education, democracy, autonomous and critical thinking.

\footnotetext{
1 Paris Catholic University, Education Faculty: 3 rue de l'Abbaye, 75006 - Paris, France. hugonnierb@gmail.com 


\section{Education et démocratie}

La démocratie n'est pas, loin s'en faut, le régime politique qui prévaut unilatéralement dans le monde (voir tableau ci-dessous). Pourtant, personne de nos jours ne remet en cause le fait que la démocratie soit le meilleur de tous les régimes politiques pour garantir l'avancée et la protection de la liberté.

Table 1. Répartition des régimes démocratiques dans le monde

\begin{tabular}{|l|c|c|c|}
\hline Régimes & Nombre de pays & $\begin{array}{c}\text { Pourcentage de } \\
\text { pays dans le monde }\end{array}$ & $\begin{array}{c}\text { Pourcentage de la } \\
\text { population dans le } \\
\text { monde }\end{array}$ \\
\hline Démocratie complète & 25 & 15 & 11,3 \\
\hline Démocratie partielle & 53 & 31,7 & 37,1 \\
\hline Démocratie hybride & 37 & 22,2 & 14,0 \\
\hline Régime autoritaire & 52 & 31,1 & 37,6 \\
\hline Total & 167 & 100 & 100 \\
\hline
\end{tabular}

Source : http://www.actualitix.com/democraties-dans-le-monde.html (données de 2012)

Dans tous les pays, historiquement, la démocratie a progressé lorsque le niveau général de l'éducation de la population a fait de même et inversement. C'est ce qui a permis à la population, comme le notait Condorcet, de faire davantage confiance à sa raison plutôt qu'à ceux qui détiennent les rênes du pouvoir, et de faire ainsi reculer l'obscurantisme. De fait, il faut des hommes éduqués pour participer à la délibération commune et voter ; de même qu'il faut des hommes éduqués pour assumer les responsabilités du pouvoir.

Dans cet article, nous allons nous adresser en conséquence à trois questions : en premier lieu, quelle est la relation précise entre démocratie et éducation dans les temps présents ? En second, que nous apprennent les résultats de la dernière enquête internationale PISA sur la situation de la démocratie dans les pays les plus avancés (soit l'Occident) ? Enfin, que nous révèlent les récents résultats des élections aux Etats-Unis, de nouveau, sur la situation de la démocratie mais aussi sur les besoins en termes d'éducation?

\section{Relation entre démocratie et éducation}

Plus un peuple est éclairé par la vérité, plus il est libre et plus les chances de chacun de réussir sont égales, plus la démocratie avance. L'éducation est donc indispensable car sans elle la vérité ne peut s'affirmer, les hommes restant ignorants ne sont pas libres et l'égalité des chances ne peut prévaloir. Ainsi, plus la connaissance, la liberté et la justice cheminent, grâce à l'éducation, plus les démocraties progressent.

Les grands auteurs de la philosophie politique (Hobbes, Locke, Rousseau et Kant) ont conceptualisé l'idée d'un contrat social que la société civile aurait signé avec l'État naissant et dont les termes auraient été une perte pour chacun de liberté - qui prévalait à l'état de nature faite de violences, de guerres, et d'injustices - contre la sécurité, la paix et la justice offertes par l'État qui aurait le monopole de la violence. Dans ce contrat, cependant, l'éducation n'était pas présente. Pourtant, en contrepoint de la police et de la justice, l'éducation, comme on l'a vu, facilite le développement de la liberté. Par ailleurs l'éducation, à travers l'égalité des chances, conduit à une plus haute justice sociale, ce que les corps judiciaires n'autorisent pas vraiment. Enfin seule, l'éducation permet de se rapprocher de la vérité dont sont tant épris les peuples. Ainsi, tout 
naturellement, un " second " contrat social devrait aujourd'hui être développé pour inclure l'éducation et des réflexions devraient être menées pour le conceptualiser en reprenant la problématique des philosophes contractualistes.

Mais si la démocratie est indispensable au développement de l'éducation, une éducation démocratique l'est elle-même pour le déploiement de la démocratie. Cela suppose que prévalent entre tous les citoyens une égalité d'accès à l'éducation, une égalité de qualité d'offre éducative et une égalité des chances. Or, comme l'a analysé Marcel Gauchet, tel n'est pas le cas en France. Et cela à un tel point que, selon lui, il existe un antagonisme entre la dynamique démocratique et la transmission des connaissances. En France, le niveau d'éducation est tout juste moyen et baisse avec le temps, ce qui entrave le développement de la vérité et de la liberté; selon les enquêtes PISA de l'OCDE, la taille de l'élite régresse et celle des laissés-pour-compte s'accroit ce qui risque de conduire à terme à un certain despotisme, donc à moins de liberté. Dans le même temps, les inégalités scolaires sont les plus fortes de l'OCDE et augmentent, ce qui signifie une baisse de la justice éducative et une augmentation des inégalités sociales. La démocratie risque donc de régresser en France. Si les pathologies de la démocratie dans les domaines politiques, économiques et sociaux ont été bien étudiées (voir, par exemple, Raymond Aron et Cynthia Fleury), il est temps d'étudier de plus près les pathologies de l'éducation pour faire avancer la démocratie.

Par ailleurs, la démocratie ne peut se développer sans une forte éducation à la démocratie. Quels sont les valeurs et les principes fondateurs de la démocratie que l'on doit enseigner? Liberté et égalité (l'isonomie) viennent évidemment en tout premier lieu car même si les hommes ne naissent pas tous égaux et libres, c'est le propre des institutions démocratiques de les rendre tels. Mais on peut citer ensuite la tolérance puisque l'Histoire a montré que c'est toujours grâce elle que l'isonomie a progressé. Autre question d'importance, quel type de citoyens les systèmes éducatifs doivent-ils former? Par exemple, on peut distinguer, suivant en cela Denis Meuret, deux modèles : celui de l'américain John Dewey, philosophe, qui cherche à construire une école adaptée à une société démocratique, une école libre, apte à se transformer rapidement, un laboratoire de citoyenneté favorisant l'action sociale et politique, formant des hommes libres. Et celui du français Emile Durkheim, sociologue, qui cherche à construire une école suivant une forme sociale idéale, donc plus rigide, formant des citoyens modèles pour servir une République modèle, favorisant l'intégration politique et sociale. Ces paradigmes sont-ils encore pertinents ? A l'ère de la mondialisation, de l'économie de la connaissance et du numérique, ne faut-il pas en penser d'autres ? Suivant quels critères et quelles valeurs ? Pour quelle démocratie ? Enfin, pour survivre, la démocratie n'a-t-elle pas besoin de citoyens disposant d'un fort esprit critique et de grandes capacités d'analyse, de discernement et de jugement pour ne pas être trop influencés par les diverses idéologies et démagogies et pour savoir développer leur propre interprétation du monde et de la société ?

D'autre part, à l'ère de la mondialisation, la démocratie ne peut plus guère être enfermée dans un seul territoire; c'est le cas notamment en Europe. II faut donc apprendre aux jeunes de tous les pays européens ce que sont les valeurs et les principes que défend l'Union Européenne, leur expliquer comment les institutions de l'U.E. sont elles-mêmes démocratiquement instituées et pourquoi appartenir à cette union renforce et protège la démocratie des pays participants.

Enfin, on ne peut que s'interroger, au vu du tableau ci-dessus, sur le nombre encore important de régimes autoritaires et en conséquence sur le niveau d'éducation des populations de ces pays. Il est en effet très vraisemblable que, dans la plupart d'entre eux, les populations sont à dessein 
sous-éduquées afin que la population accorde la plus grande importance aux dires du ou des gouvernants et que la moindre aspiration vers de plus grandes libertés soit restreinte au maximum.

\section{PISA et la démocratie en Occident}

Selon l'OCDE (2016), les pays de l'OCDE ont continué à investir dans l'éducation et pourtant cela ne s'est traduit que rarement par des améliorations sensibles des performances des élèves. En effet, de 2012 à 2015, les élèves, qui ont vu leurs performances augmenter, étaient ceux des pays en développement. Dans les pays de l'OCDE, les performances ont eu tendance à stagner ou à régresser, avec quelques exceptions comme au Chili, au Danemark, en Espagne, en Israël, en Norvège, en Pologne et au Portugal. Ceci résulte du fait que, dans les pays occidentaux, le pourcentage moyen d'élèves en grande difficulté, qui sont souvent issus des classes défavorisées, ne diminue pas, au contraire. Ce pourcentage s'élevait à 17,7 \% en sciences en 2012. II est désormais de $21,3 \%$, soit une augmentation de $20 \%$. Dans le même temps, le pourcentage moyen d'élèves très performants, soit l'élite, a diminué, passant de $8,4 \%$ à $7,7 \%$, une baisse en trois ans de $8,3 \%$. Le premier phénomène pose un problème social crucial évident; le second un problème économique d'importance, les deux phénomènes réunis un problème politique majeur.

Avec la hausse des jeunes en grande difficulté, il s'ensuit, selon l'OCDE, que dans les pays développés, les élèves issus de classes défavorisées ont aujourd'hui 2,8 fois plus de probabilités que les élèves de classes favorisées de ne pas atteindre le niveau minimum de performances en sciences. Il y a donc de grands risques pour eux de quitter le système éducatif sans aucun diplôme, ce qui va entraver durablement leurs chances de succès non seulement dans leur vie professionnelle, mais aussi sociale et personnelle. De plus, les jeunes issus de l'immigration ont en moyenne des performances inférieures à celles des autres élèves, même dans le cas où ils sont de deuxième génération et qu'ils ont donc fait la totalité de leur scolarité dans le même pays. Ceci pointe l'intégration scolaire insuffisante de ces jeunes, ce qui aggrave la situation sociale et dilue le lien social entre les communautés. L'OCDE explique en partie cet écart de performances par la ségrégation résidentielle. En effet, une fois pris en compte le statut socio-économique des élèves, cet écart de performances s'amenuise ou même disparaît, ce qui montre qu'il est davantage expliqué par le déterminisme social que par de moindres capacités des élèves. II faut donc développer rapidement une école plus équitable et plus inclusive en lui en donnant réellement et durablement les moyens.

Avec la baisse du pourcentage d'élèves très performants, c'est à terme une baisse $d u$ pourcentage d'élèves qui vont rejoindre les filières scientifiques et techniques. Ceci ne peut qu'avoir un impact sur la recherche, le développement et l'innovation en général, et, partant, sur la compétitivité des entreprises et des économies. II convient donc de redonner de l'ambition à l'éducation et d'apporter un souffle nouveau à l'enseignement des matières scientifiques.

Enfin, la simultanéité des deux phénomènes laisse craindre un impact politique négatif : la baisse du pourcentage d'élèves très performants peut mener à terme à une réduction de l'élite des pays, de sorte que les pouvoirs de toutes sortes seront détenus dans un nombre de mains plus limité. Rien n'est pire pour la démocratie. De même, il est inquiétant pour la démocratie que le nombre de personnes à même de bien maîtriser leur destin s'amenuise (avec l'augmentation du pourcentage de jeunes qui, à 15 ans, rencontrent de grandes difficultés à l'école, ce qui laisse présager qu'il en sera de même plus tard). Cela fait craindre une dérive inquiétante vers un certain despotisme. La défiance actuelle à l'égard des élites s'expliquerait-il par cela ? On sait les démocraties fragiles car quelques pathologies les guettent. L'éducation est la meilleure arme de 
construction et de protection de la démocratie. Les contre-performances mises en avant par PISA montrent que l'éducation doit faire l'objet de mesures visant à améliorer rapidement son efficacité.

\section{Les élections aux Etats-Unis et l'éducation}

A de maints égards, le 45ème président des Etats-Unis inquiète, que ce soit en ce qui concerne sa politique internationale, ses projets économiques, fiscaux et financiers, ses intentions en matière d'immigration ou encore ses propos sur les femmes. Mais il nous inquiète aussi car, sans qu'on veuille vraiment le reconnaître, on craint avec lui la montée d'un certain totalitarisme. Ce dernier a été longuement examiné par Hannah Arendt dans « Les origines du totalitarisme ». Il est peut-être utile d'analyser à cette lumière la situation américaine actuelle.

Un des premiers éléments, qui caractérise le totalitarisme, c'est la montée d'un mouvement de masses constituées pour l'essentiel d'individus rarement membres d'un parti, souvent socialement isolés et qui se sentent soit spoliés, soit défavorisés, soit encore déclassés ou déconsidérés dans la société actuelle. Ils font certes très souvent partie des classes populaires, mais on trouve également des individus au sein de l'élite qui se considèrent aussi comme des laissés-pour-compte du système. Tous sont contre 'l'establishment', raillent les politiciens, se moquent de la respectabilité des chefs et n'ont rien à perdre si le système explose. Cette sorte d'alliance entre les masses et une partie de l'élite est le second facteur important qui caractérise la montée du totalitarisme.

Le troisième facteur est évidemment l'arrivée opportune d'un leader qui stigmatise la classe politique, remet en cause l'ensemble de ses objectifs, casse les codes moraux, impose de nouvelles postures, se moque des gens établis y compris dans son propre parti, et concentre sa vindicte sur une partie de la population. Cette attitude a un effet dynamiseur sur les masses qui voient l'arrivée de ce leader comme l'opportunité d'une "revanche » et qui, en conséquence, adhérent totalement à tout ce qu'il dit et fait, même si, et peut-être surtout, lorsque c'est excessif. Le leader est ainsi paré d'une aura d'infaillibilité, un des faits saillants du leader totalitaire qui en arrive à énoncer, sans sourciller, des contre-vérités devant des millions de téléspectateurs. Autre propriété du totalitarisme, sur la base d'une science que lui seul connaît, ses sources statistiques étant invérifiables (de sorte que ce qu'il dit relève de la propagande), le leader promet pour l'avenir un nouveau paradis ; selon le leader, l'avenir sera toujours plus radieux aussi bien pour le pays, qui deviendra à nouveau le plus fort et le plus dynamique du monde, que pour les masses. Ces promesses sont basées évidemment sur une idéologie comme tout totalitarisme; avec le 45ème président des Etats-Unis, c'est celle du « deal » : à savoir que « nous américains nous imposerons la loi du plus fort, c'est-à-dire la nôtre ».

La réponse à apporter est à travers l'éducation. Non pas pour tenter de démontrer à ceux qui suivent le leader charismatique qu'ils se trompent et font fausse route - cette approche est d'emblée condamnée tel étant l'aveuglement des masses - mais pour travailler à une longue et patiente œuvre visant à élever le niveau d'éducation de ces populations afin qu'elles puissent, à terme, développer leur discernement, s'élever dans la société et y trouver un emploi de meilleure qualité et mieux rémunéré. C'est certes une œuvre de longue haleine mais c'est une œuvre indispensable pour l'avenir de ce pays. 


\section{Conclusion}

L'éducation est le ciment de la démocratie. Sans elle, il n'y a ni progrès social, ni classe moyenne qui s'élève, ni recherches, ni innovations. Sans elle, il n'y a pas non plus de culture qui enrichit les hommes et leur vie en créant des liens entre eux et le bonheur de construire ensemble le bien commun, en dû respect des droits de chacun. Education et démocratie sont ainsi indispensables pour le progrès de l'homme et de la société.

\section{Références}

Arendt, H. (1972). Le système totalitaire. Paris : Le Seuil.

Aron, R. (1997). Introduction à la philosophie politique. Paris : Le Livre de poche.

Fleury, C. (2009). Les pathologies des démocraties. Paris : Le Livre de Poche, Biblio-Essais.

Condorcet, N. de (1791). Cinq mémoires sur l'instruction publique. Retrieved from http://classiques.uqac.ca/classiques/condorcet/cinq_memoires_instruction/Cinq_memoires _instr_pub.pdf.

Dewey, J. (1990). Démocratie et éducation. Paris : Armand Colin.

Gauchet, M. (2002). La démocratie contre elle-même. Paris : Gallimard.

Hobbes, T. (2000). Le Léviathan. Paris : Le Livre de poche.

Kant, E. (1993). Fondements de la physique des mœurs. Paris : Le Livre de poche.

Locke, J. (1993). Traité du gouvernement civil. Paris : Flammarion.

Meuret, D. (2007). Gouverner l'école: une comparaison France - Etats-Unis. Paris : Presses Universitaires de France.

OCDE/PISA (2016). Résultats du PISA 2015, OCDE. Retrieved from: https://www.oecd.org/pisa/pisa-2015-results-in-focus-FR.pdf.

Rousseau, J.-J. (2011). Le contrat social. Paris : Le Livre de Poche.

Fecha de recepción: 26/01/2017

Fecha de aceptación: 15/12/2017 